\title{
Huge Subdural Empyema with Unusual \\ Presentation in Infant
}

- Case Report-

\author{
Jun-ichiro HAMADA, Homare ICHIMURA and Yukitaka USHIO
}

Department of Neurosurgery, Kumamoto University Medical School, Kumamoto

\begin{abstract}
An 11-month-old boy presented with a huge subdural empyema in the left hemisphere associated with a subsequent contralateral chronic subdural hematoma. Burr-hole drainage and antibiotic therapy achieved a successful outcome. Subdural empyema may result from hematogenous seeding of infection into a pre-existing chronic subdural hematoma.
\end{abstract}

Key words: infant, subdural empyema, subdural hematoma

\section{Introduction}

Subdural empyema is a rare infection characterized by the collection of purulent matter in the subdural space. It is usually associated with otorhinologic infection, but can occur after trauma and intracranial surgery. Bacteremic seeding to the subdural space is rare, as only a few cases have been documented or suspected. ${ }^{1-3)}$

We report the case of an 11-month-old infant with a huge subdural empyema containing both blood and purulent material associated with Hemophilus influenza infection.

\section{Case Report}

An 11-month-old boy was the third child of healthy parents, with no history of otorhinologic disease or developmental delay. He developed headache and high fever followed by a generalized convulsion. After admission to another hospital, he was transferred to our department on the 5th day of illness.

On admission, no neck stiffness was present. His temperature was $39^{\circ} \mathrm{C}$ and head circumference was $52 \mathrm{~cm}$, much larger than normal. The anterior fontanel was slightly tensive. General physical examination found no other abnormalities. He was irritable, but with no consciousness disturbance. Neurological examination found a very mild right hemiparesis. Laboratory tests revealed a peripheral white blood cell count of $15,700 / \mathrm{mm}^{3}$, C-reactive protein (CRP) level of $10.12 \mathrm{ng} / \mathrm{ml}$, and normal urinalysis results and chemistry profile.

Skull roentgenograms demonstrated mild widening of the sutures, but neither sinusitis nor osteomyelitis. Computed tomographic scans showed a slight low-density subdural collection of fluid in the left hemisphere with rim enhancement and marked mass effect. Magnetic resonance (MR) imaging revealed a slight low-intensity area on the $\mathrm{T}_{1}$-weighted image which was high-intensity area on the $T_{2}$-weighted image, and prominent sulci and enlarged ventricle in the opposite (right) hemisphere. $\mathrm{T}_{1}$-weighted MR imaging enhanced with gadolinium-diethylenetriaminepenta-acetic acid (Gd-DTPA) disclosed a multiloculated collection of fluid surrounded by a thick, rim-enhancing area (Fig. 1). Lumbar puncture was not carried out because of the risk of cerebral herniation.

On the day of admission, a left parietal burr hole demonstrated that the epidural space was normal. Subdurally, there were a thick outer membrane and a large amount of bloody, yellow, purulent material, about $25 \mathrm{ml}$ of which was removed. The subdural

Received January 27, 1992; Accepted June 17, 1992

Author's present address: J. Hamada, M.D., Department of Neurosurgery, National Cardiovascular Center, Suita, Osaka, Japan. 

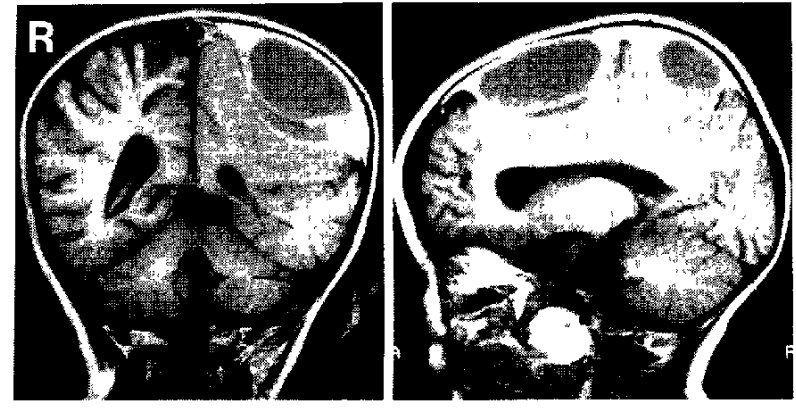

Fig. 1 Coronal (left) and sagittal (right) MR images enhanced with Gd-DTPA, demonstrating a multi-loculated subdural collection of fluid 5 days after onset.

space was irrigated with dilute bacitracin solution through a silastic catheter to avoid traumatizing the arachnoid mater until the effluent was clean. The catheter was left in place for 3 days, and removed when the drainage diminished. Culture of the material from the subdural space revealed Hemophilus influenza. Microscopic examination of the membrane revealed spotty infiltration of polymorphonuclear cells in the fibrous tissue (Fig. 2 upper).

He received penicillin $G$ and a second-generation cephalosporin intravenously for 3 weeks. After the 10 th day, his temperature was stable below $37^{\circ} \mathrm{C}$, CRP was $1.37 \mathrm{ng} / \mathrm{dl}$, and he began to show slow and steady improvement. Neurological examination revealed no abnormalities. Otolaryngologic and cardiologic consultations discovered no paranasal sinus, otogenic or congenital heart disease. $T_{1}$-weighted MR imaging 7 weeks after the initial operation showed a slight low-intensity area in the left parietal region with no mass effect and a small high-intensity area in the right frontal subdural space (Fig. 3 left). One week later, he was discharged in apparently good health and closely followed as an outpatient.

After 4 months he was well, but MR imaging showed a high-intensity area on both $T_{1^{-}}$and $T_{2}$-weighted images in the right frontoparietal subdural space with a mild mass effect. The diagnosis was an enlarged chronic subdural hematoma. The subdural empyema in the left hemisphere had disappeared (Fig. 3 center). On readmission, he was asymptomatic. Three days later, a burr hole was made and the hematoma cavity was evacuated and irrigated because of the mass effect and possibility of development into subdural empyema. Microscopic examination showed that the outer membrane had the typical appearance of connective tissue with blood vessels
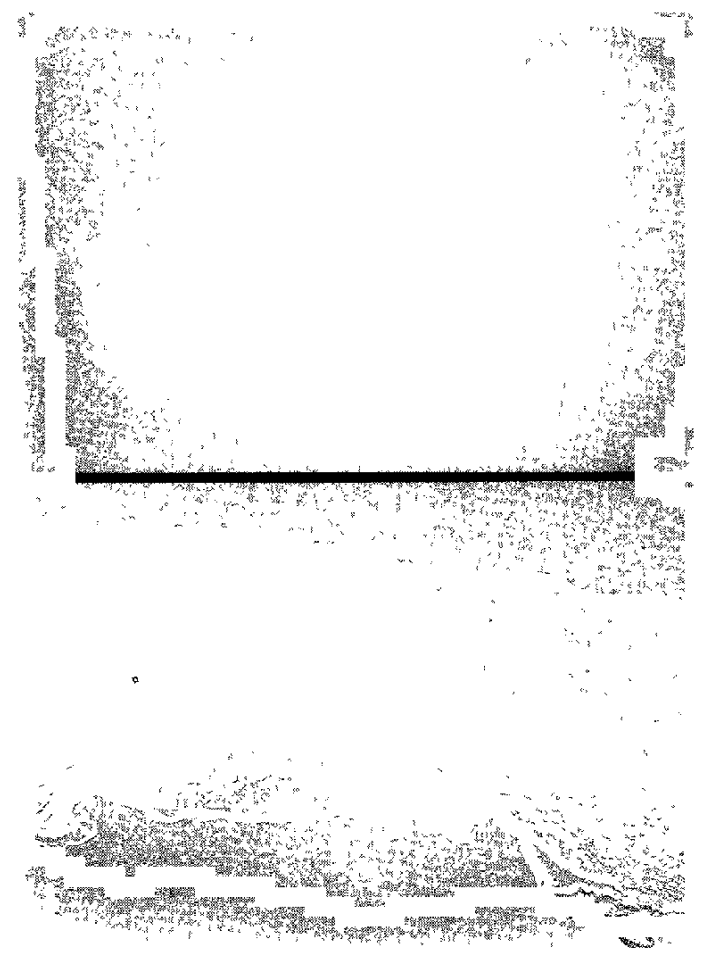

Fig. 2 Photomicrographs of the membrane obtained at the initial (upper) and second (lower) operations, revealing spotty infiltration of polymorphonuclear cells in the fibrous tissue (upper) and well-vascularized granulation tissue and collagen (lower). HE stain, upper: $\times 60$, lower: $\times 40$.

(Fig. 2 lower).

He was discharged 2 weeks later. MR imaging 1 month after the second operation demonstrated a small residual collection of fluid on the right convexity and resolution of the mass effect (Fig. 3 right). After 15 months, he appeared to be well.

\section{Discussion}

In recent years, the mortality among patients with subdural empyemas has significantly diminished, mainly due to rapid diagnosis, aggressive antibiotic therapy, and timely surgical intervention. However, subdural empyema is a neurosurgical emergency which may be fatal unless recognized and managed promptly.

The patient population falls into two age groups, infants below 2 years and adolescents between 12 and 16 years. ${ }^{4}$ The two age groups often present with a different combination of signs and symptoms. Fever and headaches are the most frequent com- 

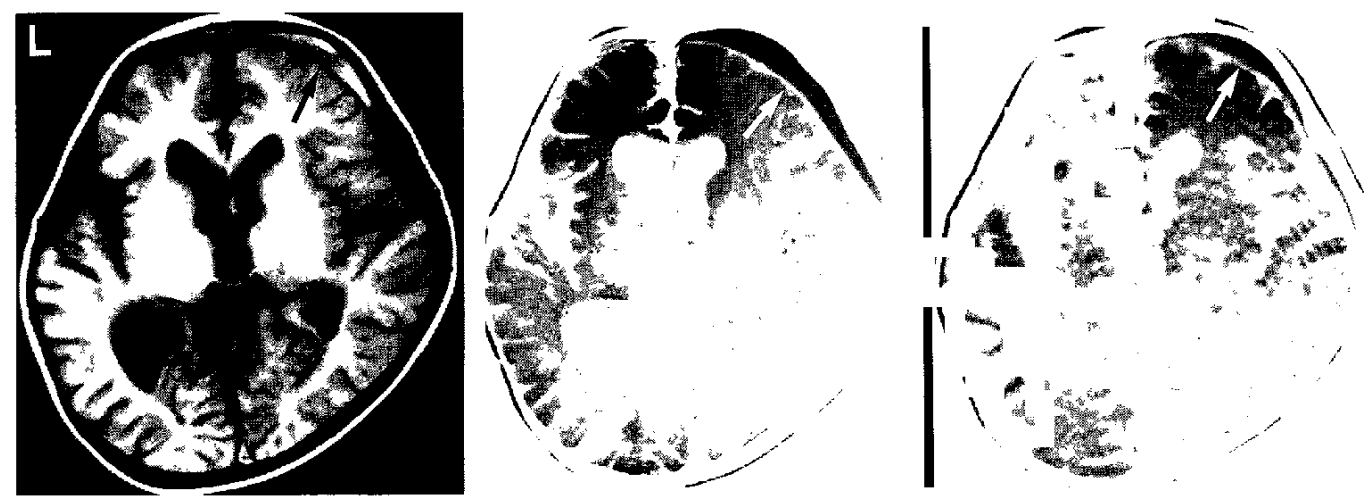

Fig. 3 left: $\mathrm{T}_{1}$-weighted MR image 7 weeks after the initial operation, showing a small high-intensity area (arrow) in the right frontal subdural space. center: $\mathrm{T}_{1}$-weighted $\mathrm{MR}$ image on readmission, showing a high-intensity collection of fluid (arrow) in the right frontoparietal space with a mild mass effect. The subdural empyema in the left hemisphere had disappeared. right: $\mathrm{T}_{1}$-weighted $\mathrm{MR}$ image 1 month after the second operation, showing a small residual chronic subdural hematoma (arrow) on the right convexity.

plaints in infants. Infants often present with signs and symptoms similar to meningitis, while seizures and focal neurological deficits occur more frequently in adolescents.

The most common source of subdural empyema is a direct extension of otitis media or sinusitis into the subdural space. In children, spread of pneumococcal meningitis from the subarachnoid to the subdural space is also frequent. A less common cause is iatrogenic infection of a subdural hematoma. Subdural empyema resulting from hematogenous seeding of infection into a pre-existing subdural hematoma is very rare. Genest et $a .^{2)}$ described a 35 year-old male with bilateral subdural empyema probably introduced into the subdural space via the blood stream from the urinary tract. Coonrod and Dans" reported a subdural empyema secondary to bacteremic seeding from a chronic subdural hematoma occurring in a 53-year-old male. Pattisapu and Parent ${ }^{3)}$ described eight children with subdural empyemas, and suggested hematogenous seeding of pre-existing chronic subdural hematomas as the cause of bilateral parietal subdural empyemas in a 2-month-old infant. However, no further details on the etiology were given.

In our patient, the subdural empyema was associated with a subsequent contralateral chronic subdural hematoma. The subdural fluid obtained at the first operation consisted of purulent matter and blood, and there was no history of otorhinologic or cardiologic disease. These features suggest that the subdural empyema originated from a pre-existing chronic subdural hematoma which became infected.
If our case had been a common subdural empyema, severe damage would have resulted. However, he only presented with a very mild hemiparesis, suggesting that an inner membrane protected the surface of the brain from pus.

Weingarten et al ${ }^{55}$ presented MR images of six patients with extra-axial empyemas which demonstrated convexity and interhemispheric collections of fluid appearing slightly hyperintense relative to cerebrospinal fluid (CSF) and hypointense relative to white matter on $T_{1}$-weighted images, and hyperintense relative to both CSF and white matter on $\mathrm{T}_{2}$-weighted images. After 1-3 weeks, contrast medium caused rim enhancement, believed to indicate the formation of a capsule around the pus. These features are consistent with our case.

The most common organisms causing subdural empyema in infants are Hemophilus influenza and aerobic Streptococcus. A combination of penicillin $\mathrm{G}$ and a second- or third-generation cephalosporin is effective against these organisms and should be continued for at least 3 weeks.

Surgical management of subdural empyemas is controversial, but in our case, the placement of a burr hole and catheter drainage was effective.

The possible development of a chronic subdural hematoma into a subdural empyema should be considered when managing pediatric patients.

\section{References}

1) Coonrod JD, Dans PE: Subdural empyema. $A m J$ Med 53: 85-91, 1972 
2) Genest CAS, Bingham MWG, Hamilton LCRD: Bilateral subdural empyema: Report of a case with arteriograms. J Neurosurg 20: 524-526, 1963

3) Pattisapu JV, Parent AD: Subdural empyemas in children. Pediatric Neuroscience 13: 251-254, 1987

4) Smith HP, Hendrick EB: Subdural empyema and epidural abscess in children. J Neurosurg 58: 392-397, 1983

5) Weingarten K, Zimmerman RD, Becker RD, Heier
LA, Haimes AB, Deck MDF: Subdural and epidural empyemas. MR imaging. AJR 152: 615-621, 1989

Address reprint requests to: J. Hamada, M.D., Department of Neurosurgery, National Cardiovascular Center, 5-7-1 Fujishirodai, Suita, Osaka 565, Japan. 